\title{
Opioids in Canada
}

Vera Grywacheski, Allison Sabad, Lyubov Kushtova, Michele Bender, Nicole Rennie, Roger Cheng and Krista Louie

\begin{abstract}
The opioid crisis in Canada is a complex issue with devastating consequences for individuals, families and communities. Pan-Canadian data that provide a better understanding of the crisis are a high priority to help guide efforts for reducing opioid-related harms. The objective of this study is to summarize recent trends of opioid-related harms and opioid prescribing across Canada. Opioid-related harms examined in this study include opioid poisonings, opioid use disorders, adverse reactions to opioids and infants born with neonatal withdrawal symptoms. Data are reported at the pan-Canadian, provincial and community levels.
\end{abstract}

\section{Approach}

Data from the Canadian Institute for Health Information's (CIHI) Hospital Morbidity Database, Ontario Mental Health Reporting System and National Ambulatory Care Reporting System were used to identify opioid-related hospitalizations and emergency department (ED) visits across Canada between 2013 and 2017. The ED analysis was based on available, comparable data at the time of the study and included visits from Ontario, Alberta and Yukon. This analysis focused on confirmed cases of opioid-related harms and excluded suspected diagnoses. Facility postal codes were used for the provincial analysis, and patient postal codes were used for the community-level analysis by census subdivision, limited to populations of 50,000 or more.

CompuScript data were obtained under licence from IQVIA Solutions Canada Inc. to examine trends in opioid prescribing over the same period. The CompuScript database includes Canadian aggregate dispensed prescription data projected from a sample of $6,000+$ pharmacies, representing more than $60 \%$ of all retail pharmacies in Canada. Defined daily dose (DDD) calculations for opioids were based on the WHO Collaborating Centre for Drug Statistics Methodology (2018). The DDD for codeine was based on the International Narcotic Control Board standard for analgesia of $240 \mathrm{mg}$ per day (International Narcotic Control Board 2017). Methadone and buprenorphine/naloxone combinations were excluded from the analysis.
Population data from Statistics Canada were used to calculate crude and age-adjusted rates. The direct standardization process was used with the 2011 Canadian population as the reference year. $^{1}$

\section{Key Findings \\ Pan-Canadian trends for opioid-related harms and prescribing}

In recent years, many opioid-related harms have increased across Canada despite decreasing trends in the amount of opioids being prescribed to Canadians (Figure 1).

Between 2013 and 2017, rates of hospitalizations and ED visits for opioid poisonings and opioid use disorders, as well as rates of infants born with neonatal withdrawal symptoms, consistently increased each year. In 2017, the crude rates for opioid poisonings and opioid use disorders were 17 and 32 hospitalizations per 100,000 population size, respectively. These rates amounted to 6,072 opioid poisonings and 11,768 opioid use disorder hospitalizations across Canada in 2017. In the same year, more than five infants per 1,000 live births, totalling 1,908 neonates, had significant symptoms of neonatal withdrawal. These opioid-related harms may be due to the use of illicit opioids, prescribed opioids or a combination of both.

Adverse drug reactions, that is, when an opioid is taken as prescribed and results in harm, is the only category of opioidrelated harm that experienced a decrease over the study period. Between 2013 and 2017, the rate of adverse drug reactions decreased from 29 to 27 hospitalizations per 100,000 population size and from 19 to 17 ED visits per 100,000 population size. Decreases in adverse drug reactions may be as a result of a variety of initiatives and guidelines implemented during the study period aimed at reducing the risk of harms from opioid prescribing, such as early adoption of alternate prescribing guidelines and development and expansion of prescription monitoring programs in some jurisdictions (Canadian Centre on Substance Abuse 2015; College of Physicians and Surgeons of British Columbia 2016; Dowell et al. 2016; National Pain Centre 2017). 
FIGURE 1.

Trends in rates of opioid-related harms and opioid prescribing in Canada, 2013-2017
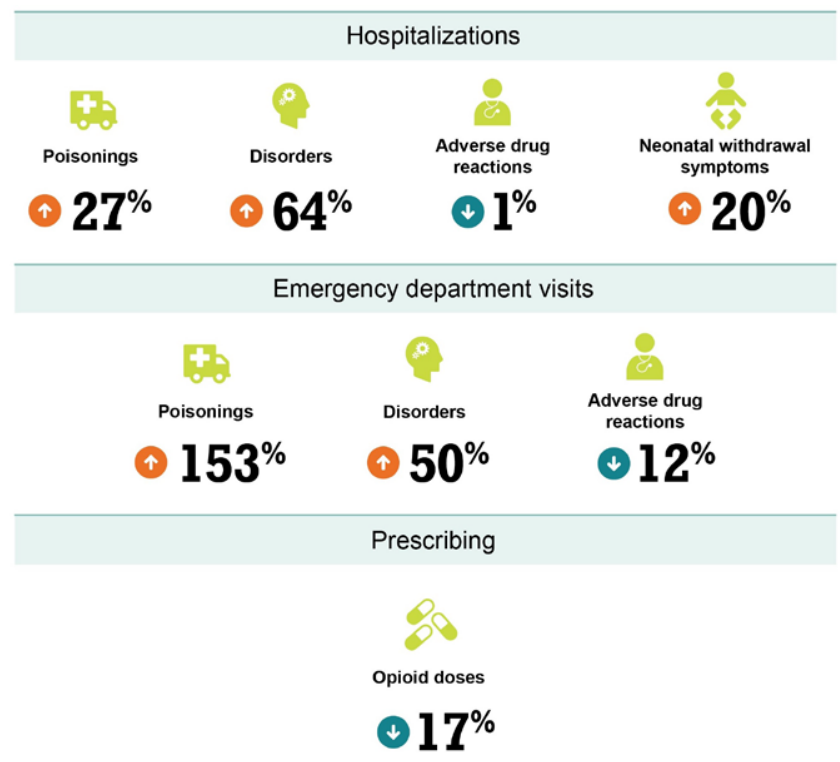

Notes

Poisoning occurs when an opioid is taken incorrectly and results in harm.

Disorders include a wide variety of mental health and behavioural disorders that are attributable to the use of opioids.

Adverse drug reaction occurs when an opioid is taken as prescribed and results in harm. Neonatal withdrawal symptoms occur when an infant experiences withdrawal symptoms from the mother's use of drugs or addiction. These include neonatal abstinence syndrome and drug withdrawal syndrome.

With the exception of adverse drug reactions, opioid-related harms can be caused by either prescription or illicit opioids.

Prescribing data exclude the territories and reflect daily defined doses per 1,000 population. Hospitalizations and emergency department visits reflect crude rate per 100,000 population. Emergency department visits are limited to Ontario, Alberta and Yukon.

Suspected cases are excluded.

Sources

Hospital Morbidity Database, Canadian Institute for Health Information; prepared using data from CompuScript, IOVIA.

The increasing rates of opioid poisonings, opioid use disorders and infants born with neonatal withdrawal symptoms across the country, despite decreasing trends in opioid prescribing, allude to the likely impact of the illicit market. In 2017, fentanyl prescriptions accounted for only $8.6 \%$ of all opioid doses (as measured by DDDs) dispensed from retail pharmacies, although fentanyl and fentanyl analogues accounted for the majority $(72 \%)$ of accidental apparent opioid-related deaths between January and September of the same year (Public Health Agency of Canada 2018). Fentanyl and fentanyl-related substances continue to be a major driver of the opioid crisis; however, data are not currently available to indicate which of these harms are due to prescription or illicit sources at the pan-Canadian level.

\section{Provincial trends for opioid-related harms and opioid prescribing}

Trends for opioid-related harms and opioid prescribing vary by province (Figure 2). Looking at the most recent data, between 2016 and 2017, the amount of opioid doses prescribed decreased in all provinces except in Newfoundland and Labrador, where prescribing increased by $2 \%$. Despite recent decreasing trends for opioid prescribing in British Columbia (-14\%), Manitoba $(-5 \%)$ and Ontario $(-12 \%)$, opioid poisonings increased by 5.1, 2.5 and 1.6 hospitalizations per 100,000 population size in each jurisdiction, respectively.

\section{FIGURE 2.}

Trends for opioid poisoning hospitalizations and opioid prescribing, Canada, 2016-2017

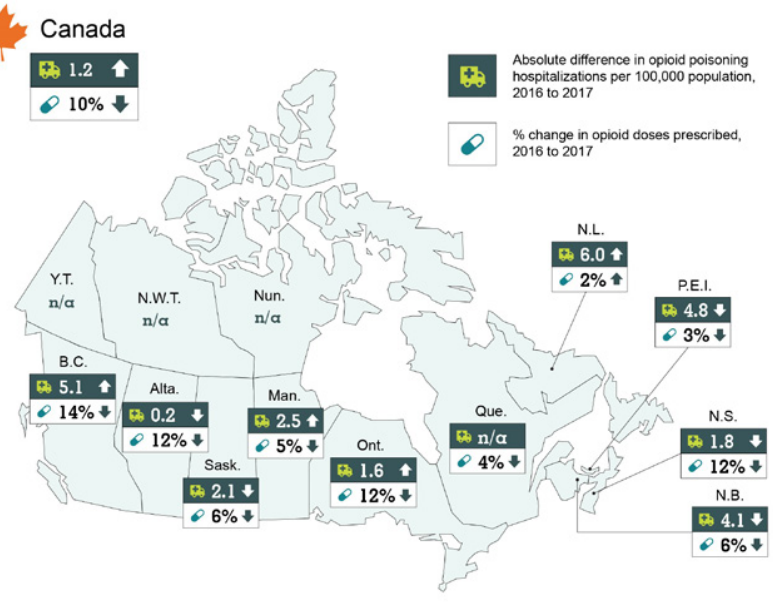

$\mathrm{n} / \mathrm{a}=$ not available.

Notes

Opioid poisoning hospitalizations reflect age-adjusted rate per 100,000 population.

Prescribed opioid doses reflect daily defined doses per 1,000 population.

Opioid poisoning hospitalizations can be caused by either prescription or illicit opioids or a combination of both.

Sources

Hospital Morbidity Database, Canadian Institute for Health Information; prepared using data from CompuScript, IOVIA.

\section{Community trends for opioid-related harms}

Opioid poisonings are affecting people from all walks of life across the country. However, smaller Canadian communities are experiencing opioid poisoning hospitalization rates that are more than double those in Canada's largest cities (Figure 3). In 2017, the highest rates of opioid poisoning hospitalizations were in communities with populations between 50,000 and 99,999.

In 2017, some of the Canadian communities with the highest rates of opioid poisoning hospitalizations by census subdivision included Nanaimo (BC), Prince George (BC), Kelowna (BC), Brantford (ON), Kamloops (BC), Belleville 
$(\mathrm{ON})$, Medicine Hat $(\mathrm{AB})$, Sault Ste. Marie $(\mathrm{ON})$ and Victoria (BC). The age-adjusted rate of opioid poisonings in these communities ranged from 40 to almost 60 hospitalizations per 100,000 population size.

An analysis of ED visits by census subdivision, limited to Alberta and Ontario, found the highest rates of opioid poisonings in Sault Ste. Marie (ON), Brantford (ON), Lethbridge $(\mathrm{AB})$, Red Deer $(\mathrm{AB})$, Niagara Falls $(\mathrm{ON})$, Welland $(\mathrm{ON})$, Peterborough $(\mathrm{ON})$, Medicine Hat $(\mathrm{AB})$ and St. Catharines $(\mathrm{ON})$. The age-adjusted rate of opioid poisonings in these communities ranged from 124 to almost 177 ED visits per 100,000 population size in 2017.

\section{FIGURE 3.}

Rates of hospitalizations from opioid poisoning by community size, Canada, 2017

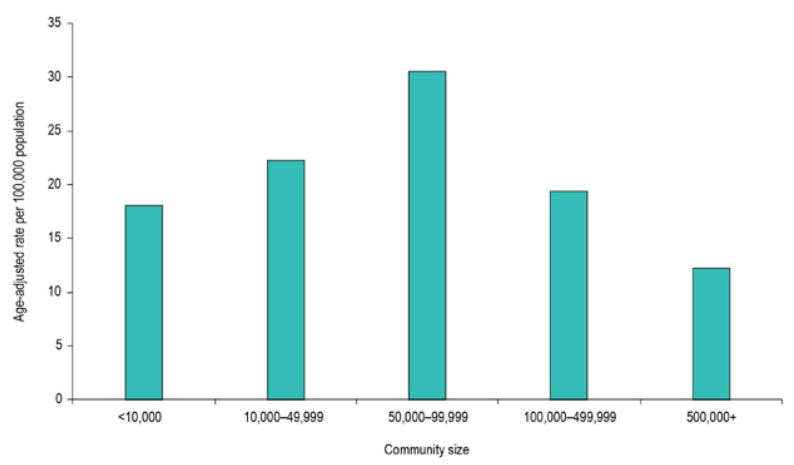

Note

Quebec data and population data are from 2016 (the most recent year of data available). Source

Hospital Morbidity Database, Canadian Institute for Health Information.

\section{Additional Findings}

Detailed findings for opioid-related harms and use across Canada, as well as more information on data sources, terminology and methods, are available at https://www.cihi.ca/en/ opioids-in-canada.

\section{Moving Forward}

Although fewer opioids are being prescribed to Canadians, the harms associated with their use are on the rise, which likely indicates the impact of illicit opioid sources across the country. More detailed pan-Canadian data that distinguish the source of opioids (illicit or licit) that resulted in harm would be valuable for further understanding this complex public health issue. HQ

\section{Note}

1. Detailed methodology is available in the following reports:

- Opioid-Related Harms in Canada: https://www.cihi. $\mathrm{ca} /$ sites/default/files/document/opioid-related-harmsreport-2018-en-web.pdf; and
- Pan-Canadian Trends in the Prescribing of Opioids and Benzodiazepines, 2012 to 2017: https://www.cihi. $\mathrm{ca} /$ sites/default/files/document/opioid-prescribingjune2018-en-web.pdf.

\section{References}

Canadian Centre on Substance Abuse. 2015. "Prescription Monitoring Programs in Canada: Best Practice and Program Review.” Retrieved January 21, 2019. <https://www.csam-smca.org/wp-content/uploads/2014/11/BSproule-Decreasing-Harm-from-Prescription-drugs.pdf>.

College of Physicians and Surgeons of British Columbia. 2016. "Safe Prescribing of Opioids and Sedatives." Retrieved January 21, 2019. $<$ https://www.cpsbc.ca/files/pdf/PSG-Safe-Prescribing.pdf>.

Dowell, D., T.M. Haegerich and R. Chou. 2016. "CDC Guideline for Prescribing Opioids for Chronic Pain - United States, 2016." MMWR Recommendations and Reports 65(No. RR-1): 1-49. doi:10.15585/ mmwr.rr6501e1.

International Narcotics Control Board. 2017. Narcotic Drugs: Estimated World Requirements for 2018 - Statistics for 2016. Retrieved February 28, 2018. <https://www.incb.org/documents/Narcotic-Drugs/TechnicalPublications/2017/Narcotic_drugs_technical_publication_2017.pdf >.

National Pain Centre. 2017. The 2017 Canadian Guideline for Opioids for Chronic Non-Cancer Pain. Retrieved January 21, 2019. $<$ http://nationalpaincentre.mcmaster.ca/documents/Opioid $\% 20$ GL\%20for\%20CMAJ_01may2017.pdf>.

Public Health Agency of Canada, Special Advisory Committee on the Epidemic of Opioid Overdoses. 2018. "National Report: Apparent Opioid-Related Deaths in Canada (January 2016 to September 2017) Web-Based Report." Retrieved January 21, 2019. <https://www.canada. $\mathrm{ca} /$ en/public-health/services/publications/healthy-living/nationalreport-apparent-opioid-related-deaths-released-march-2018.html>.

WHO Collaborating Centre for Drug Statistics Methodology. 2018. "ATC/DDD Index." Retrieved February 28, 2018. <https://www. whocc.no/atc_ddd_index/>.

\section{About the Authors}

This paper was prepared by the Opioid Reporting team at the Canadian Institute for Health Information (CIHI). Inquiries can be sent to help@cihi.ca.

Vera Grywacheski, BA (Hons), MPH, is a senior analyst at $\mathrm{CIHI}$, Ottawa, ON.

Allison Sabad, BHSc, MHS, is a senior analyst at CIHI, Ottawa, ON.

Lyubov Kushtova, BSc (Hons), $\mathrm{MPH}$, is an analyst at $\mathrm{ClHI}$ Toronto, ON.

Michele Bender, BScPharm, MBA, is a program consultant at $\mathrm{CIHI}$, Ottawa, ON.

Nicole Rennie, BPH (Hons), is a co-op student at $\mathrm{CIHI}$, Ottawa, ON.

Roger Cheng, BScPharm, PharmD, is a senior analyst at $\mathrm{ClHI}$, Toronto, ON.

Krista Louie, BSc, MSc, is a manager at $\mathrm{ClHI}$, Ottawa, ON. 